\title{
MRI and ${ }^{1 H}$ MRS of The Breast: Presence of a Choline Peak as Malignancy Marker is Related to k21 Value of the Tumor in Patients with Invasive Ductal Carcinoma
}

\author{
Patricia R. Geraghty, MD, Maurice A.A.J. van den Bosch, MD, PhD, Daniel M. Spielman, \\ PhD, Sandeep Hunjan, PhD, Robyn L. Birdwell, MD, Katherine J. Fong, MD, Lara A. Stables, \\ PhD, Marowan Zakhour, MD, Robert J. Herfkens, MD, and Debra M. Ikeda, MD \\ Department of Radiology, Stanford University School of Medicine, Stanford, California
}

\begin{abstract}
To assess which specific morphologic features, enhancement patterns, or pharmacokinetic parameters on breast Magnetic Resonance Imaging (MRI) could predict a false-negative outcome of Proton MR Spectroscopy $\left({ }^{1} \mathrm{H}\right.$ MRS) exam in patients with invasive breast cancer. Sixteen patients with invasive ductal carcinoma of the breast were prospec-tively included and underwent both, contrast-enhanced breast MRI and ${ }^{1} \mathrm{H}$ MRS examination of the breast. The MR images were reviewed and the lesions morphologic features, enhancement patterns and pharmacokinetic parameters (k21value) were scored according to the ACR BI-RADS-MRI lexicon criteria. For the in vivo MRS studies, each spectrum was evaluated for the presence of choline based on consensus reading. Breast MRI and ${ }^{1} \mathrm{H}$ MRS data were compared to histopathologic findings. In vivo ${ }^{1} \mathrm{H}$ MRS detected a choline peak in 14/16 (88\%) cancers. A false-negative ${ }^{1} \mathrm{H}$ MRS study occurred in 2/16 (14\%) cancer patients. K21 values differed between both groups: the 14 choline positive cancers had k21 values ranging from 0.01 to $0.20 / \mathrm{second}$ (mean $0.083 / \mathrm{second}$ ), whereas the two choline-negative cancers showed k21 values of 0.03 and 0.05/second, respectively (mean 0.040/second). Also enhancement kinetics did differ between both groups; typically both cancers that were choline-negative showed a late phase plateau (100\%), whereas this was only shown in 51/4 (36\%) of the choline positive cases. There was no difference between both groups with regard to morphologic features on MRI. This study showed that false-negative ${ }^{1} \mathrm{H}$ MRS examinations do occur in breast cancer patients, and that the presence of a choline peak on ${ }^{1} \mathrm{H}$ MRS as malignancy marker is related to the $\mathrm{k} 21$ value of the invasive tumor being imaged.
\end{abstract}

\section{Keywords}

breast cancer; k21-value; magnetic resonance imaging; proton MR spectroscopy

Contrast-enhanced breast magnetic resonance imaging (MRI) detects breast cancer with almost $100 \%$ sensitivity, and plays an increasingly important role in breast cancer detection and staging (1). However, the specificity of breast MRI is rather variable, ranging from $37 \%$ to $97 \%$, resulting in moderate false-positive rates and unnecessary biopsies (2-5).

To optimize specificity in breast MRI combined high spatial-resolution imaging and rapid dynamic imaging for concurrent evaluation of detailed lesion morphology characteristics and

Address correspondence and reprint requests to: Maurice A.A.J. van den Bosch, MD, PhD, Department of Radiology, Lucas MR Imaging Center, 1201 Welch Road, Stanford, CA 94305-5488, USA, or mbosch@ @tanford.edu. 
contrast-enhancement dynamics is used, and can be combined for calculation of pharmacokinetic parameters and color parametric mapping of contrast dynamics $(6,7)$. Calculation of the pharmacokinetic parameter $\mathrm{k} 21$, a redistribution rate constant that quantifies the exchange rate of the used contrast agent between the intra- and extravascular spaces, has a great overall ability to discriminate between benign and malignant breast disease on MRI, with higher $\mathrm{k} 21$ values in malignant lesions than in benign lesions (6).

Techniques that could improve the positive predictive value of breast MRI even further are of value, because it would reduce the number of lesions detected at breast MRI that would require biopsy and as a consequence improve the cost-effectiveness of this imaging technique (3).

Proton MR spectroscopy ( ${ }^{1} \mathrm{H}$ MRS) has emerged as a powerful, non-invasive method for studying tumor biochemistry and has the potential to improve the specificity of contrastenhanced breast MRI to $67-100 \%(8,9)$. Several in vivo studies proved that ${ }^{1} \mathrm{H}$ MRS discriminate between benign and malignant lesions of the breast by detecting the presence of choline metabolites, a marker of increased cell-membrane turnover in tumors (9-13). ${ }^{1} \mathrm{H}$ MRS has been reported to be a reliable method because it is based on the appearance of a single spectroscopic peak, that of phosphocholine, in the spectrum at a frequency of $3.2 \mathrm{ppm}$. (14, 15). However, results of previous studies also demonstrated that the choline levels may not be as greatly elevated in some breast cancers as they are in others, and sometimes are not detected at all, resulting in false-negative diagnoses $(7,13,15)$.

It would have important clinical consequences if these so-called false-negative cases on ${ }^{1} \mathrm{H}$ MRS exam could be identified prospectively based on the breast MRI results. This prospective study was designed to assess which specific morphologic features, enhancement patterns, or pharmacokinetic parameters could be identified on breast MRI that would predict the outcome of the ${ }^{1} \mathrm{H}$ MRS exam in patients with invasive ductal carcinoma of the breast.

\section{MATERIALS AND METHODS}

In this study, 22 patients with pathology-proven invasive ductal breast cancer were prospectively selected to undergo MRI and additional ${ }^{1} \mathrm{H}$ MRS of the breast. The study was approved by the Human Subjects Panel at our institution and informed consent was acquired from each patient. Patients were excluded from the study if they did not receive contrast for the MRI exam $(n=3)$, if technical problems occurred during the in vivo MRS study $(n=2)$, or if multiple discrete lesions occurred in the same breast $(n=1)$. About 16 patients fulfilled study criteria and are the subject of this study.

The clinical histories, reports for all previous breast-imaging studies (mammography, ultrasound) and pathology reports were collected on each patient. These data were independently reviewed as follows. All patients had film-screen mammography for available for review, 13 studies performed at our institution and three studies performed at outside facilities. The 13 analog mammograms at our facility were performed on 600T or 800T mammographic units (General Electric Medical Systems, Milwaukee, WI). All mammograms were independently reviewed on dedicated mammography alternators by two MQSA-certified radiologists (DMI, RLB) specializing in breast imaging using BI-RADS terminology. Eleven of 16 patients had a breast ultrasound available for review, 10 were performed at our institution and one performed at an outside institution. The 10 ultrasounds at our facility were performed by breast imaging radiologists using a high-frequency, multi-hertz transducer (Logiq 700 General Electric Medical Systems, Milwaukee, WI) and were reviewed independently by the two breast imaging specialists (DMI, RLB).

All 16 patients underwent contrast-enhanced MRI of the breast on a 1.5T scanner (Echospeed; GE Medical Systems, Milwaukee, WI) with a phased-array breast coil (MRI devices, 
Waukesha, WI) using combined dynamic three-dimensional (3D) spiral imaging and highspatial-resolution 3D spectral-spatial excitation magnetization transfer (3DSSMT) imaging protocol previously described (6). Briefly, after an intravenous contrast bolus administration, 3D spiral scans were acquired water-specific dynamic images during the "wash-in" phase of contrast enhancement followed immediately by 3DSSMT high-spatial resolution scanning, with subsequent additional dynamic imaging during the "wash-out" phase of enhancement (6). The rapid dynamic imaging protocol employed a T1-weighted 3D spoiled gradient-echo spiral pulse sequence over a unilateral, whole breast in 32 sagittal sections (repetition time 38 milliseconds, echo time 12.3 milliseconds, $40^{\circ}$ flip angle, 20 interleaved spirals, $4.5-6 \mathrm{~mm}$ section thickness, $188 \times 188$ equivalent matrix and a 20 -cm field of view). Water selective spectral-spatial excitation was used for fat nulling and an on-resonance 1-2-1 binomial magnetization transfer pulse sequence was used for suppression of signal from normal fibroglandular tissue. This sequence repeatedly imaged the entire breast every 10.64 seconds and the 3D spiral acquisition was repeated 20 times for a dynamic series of 213 seconds. Forty seconds after starting the dynamic spiral imaging an intravenous bolus of $0.1 \mathrm{mmol} / \mathrm{kg}$ gadopentetate dimeglumine (Magnevist; Berlex Laboratories, Wayne, NJ) was injected at a rate of $2.5 \mathrm{~mL} / \mathrm{second}$. A sagittal high-spatial resolution imaging was performed immediately after the initial dynamic wash-in series. Suppression of fat and fibroglandular tissue signals were performed using 3D spectral-spatial excitation and a magnetization transfer pulse (3DSSMT), respectively. The following parameters were used: repetition time 33, echo time $9,50^{\circ}$ flip angle, matrix $512 \times 192,64$ sections, $1.5-2-\mathrm{mm}$ section thickness, 20 -cm field of view, and acquisition time of 6 minutes, 31 seconds.

For in vivo ${ }^{1} \mathrm{H}$ MRS studies, the lesions were evaluated with either a single (14 patients) or multiple voxel (two patients) in vivo PRESS (point resolved spectroscopy) MRS pulse sequence optimized for detecting choline $(16,17)$. We achieved enhanced lipid and water suppression using Band Selective Inversion with Gradient Dephasing (BASING) that consists of a frequency selective RF inversion pulse surrounded by spoiler gradient pulses of opposite signs which dephase stopband resonances and minimally affect pass-band resonances (17). The parameters used were as follows: TE/TR 144/2000 milliseconds, FOV $40 \mathrm{~cm}$, slice thickness $15 \mathrm{~mm}, 16 \times 16$ array chemical shift imaging CSI (CSI) grid with $1.5 \mathrm{~cm}^{3}$ voxels. For single voxel studies, voxel volumes ranged from a minimum of $4.5 \mathrm{cc}$ to approximately $6.0 \mathrm{cc}$ depending on the size of the tumor. The diagnostic MRI and experimental MRS sequences resulted in a 90-minute total scan time.

Spectra were processed using a SAGE/IDL spectro-scopic processing and display package (GE Medical Systems, Milwaukee, WI) running on a PC in a Linux environment. Data from the two channels acquired per breast from the phased array coil were processed separately $(3-\mathrm{Hz}$ exponential filter, Fourier transformed and phased) and weighted sum spectra were produced by combining the two channels after weighting them according to the water signal strength in nonsuppressed spectra. Chemical shifts were referenced to water at $4.7 \mathrm{ppm}$. Two separate groups of readers evaluated the MR spectra and MRI images. For the in vivo MRS studies, two spectroscopists (DS, SH) and one radiologist (DMI) evaluated each spectrum for the presence of choline as a consensus reading. All three readers were blinded to the final histopathology and MRI results. A spectrum was considered "positive" for choline if after baseline corrections there was a well-defined peak at $3.2 \mathrm{ppm}$ greater than two times the standard deviation of the baseline noise as determined from a peak-free region of the spectrum. The MRS result was considered "negative" if there was no peak at $3.2 \mathrm{ppm}$ with appearance of adequate lipid suppression and shimming.

For the MRI readings, three different radiologists (DMI, BLD, RLB) independently reviewed the contrast-enhanced MRI studies using special software for concurrent analysis of both highresolution MRI morphologic detail and contrast-enhancement dynamics on workstations 
(General Electric Medical Systems, Milwaukee, WI). The reviewers were blinded to the spectroscopy results and final histopathologic diagnosis in all patients. Lesion morphology, i.e., size, type, shape, border, and internal enhancement characteristics were recorded for each case using morphology descriptors standardized by the American College of Radiology (ACR) Breast MRI Lexicon (18). The dynamic enhancement curves were computer generated (signal intensity as a function of time) and the contrast uptake and curve type patterns were visually assessed by each of the three readers. Each enhancement curve was judged as having rapid or slow contrast uptake and the late phase curve type was determined by the curve shape 2 minutes after contrast injection or when the curve shape started to change (flat, persistent, plateau, or washout). The enhancement curve shape was rated suspicious when there was a rapid initial contrast uptake followed by washout or plateau in contrast enhancement (3).

With parametric calculations, the dynamic 3D spiral enhancement data were fitted to the twocompartment pharmacokinetic model on a pixel-by-pixel basis using custom software (6). The proportionality constant, $\mathrm{k} 21$ is a pharmacokinetic parameter redistribution rate constant that quantifies the exchange rate of the used contrast agent between the intra- and extravascular spaces. Each high-resolution 3DSSMT pixel hue was modulated as a function of the corresponding $\mathrm{k} 21$ at the same location according to a color scheme ranging from blue $(\mathrm{k} 21=$ $0.00 /$ sec-ond) to blue-green $(\mathrm{k} 21=0.005 /$ second $)$, green $(\mathrm{k} 21=0.01 /$ second $)$, green-yellow ( $\mathrm{k} 21=0.015 / \mathrm{sec}-\mathrm{ond})$, and yellow (k21>0.02/second) (19). Using the parametric mapping technique, lesions were classified as suspicious if parametric maps revealed a green-yellow or yellow color, or if the $\mathrm{k} 21$ value was $\geq 0.015 /$ second $(6,7)$. A score for the overall likelihood of cancer was assessed for each lesion based on the combined lesion morphology, contrast dynamics, and k21 parametric mapping on MRI and rated using the ACR Breast Imaging and Reporting Data System (BIRADS) final assessments of 1-5, with 5 representing the highest suspicion of malignancy (18).

For statistical analysis, Fisher's exact test was used to look for a significant difference in the MR imaging characteristics (morphology and enhancement kinetics) between two dichotomous groups, i.e., negative MRS result versus positive MRS result (two-sided, with a significance level of 0.05 ). The difference in mean $\mathrm{k} 21$ values according to MRS result category (negative versus positive) was tested for significance by means of Mann-Whitney test (with a significance level of 0.05).

\section{RESULTS}

The 16 patients ranged in age from $26-79$ years (mean age $=47$ years). Imaging characteristics on mammography and ultrasound are listed in Table 1. Pathology included 13 invasive ductal carcinomas (IDC), 1 IDC with mucinous features, 1 inflammatory IDC, and 1 IDC with associated invasive lobular carcinoma. On pathology the cancers ranged in size from 1.5-8.0 $\mathrm{cm}$, with a mean size of $4.1 \mathrm{~cm}$.

Magnetic resonance imaging of the breast revealed that 10/16 (63\%) IDCs presented as an irregular masses with spiculated $(n=6)$, irregular $(n=2)$, or lobulated $(n=2)$ borders (Table 2 ). The morphologic pattern of the remaining five IDC cases were an oval mass with speculated borders $(n=2)$, regional enhancement $(n=4)$ with irregular $(n=2)$, spiculated $(n=1)$ or nonspecific borders $(n=1)$. The internal enhancement patterns included one or multiple of the following features: rim enhancement in 10/16 cases (63\%), bright internal septations in 5/16 cases $(31 \%)$, central necrosis in $6 / 16$ cases $(38 \%)$, and enhancing clumps within the lesion in $3 / 16(19 \%)$ of the cases (Table 2). Contrast-enhancement kinetics showed that all 16 invasive ductal carcinomas displayed enhancement curve patterns suspicious for cancer, i.e., marked by rapid initial enhancement, followed by a late phase washout in $9 / 16$ cases $(56 \%)$ and a plateau in $7 / 16$ cases $(44 \%)$. 
Fifteen (94\%) of the 16 cancers had suspicious parametric mapping results, demonstrating high $\mathrm{k} 21$ values (predominately yellow and yellow/green) in the malignancies. The only cancer (6\% [1/16]) displaying equivocal, green parametric k21 mapping was an IDC with mucinous features which had a low $\mathrm{k} 21$ in a rim enhancement pattern, corresponding with rim enhancement seen on the high resolution MRI. $\mathrm{k} 21$ values ranged from $0.2 / \mathrm{second}$ to a low of $0.01 / \mathrm{sec}$-ond (mean k21 0.074/second). Of the 16 cancers, 14 had high $\mathrm{k} 21$ values ( $\mathrm{k} 21>0.015$ / second was considered high/suspicious for cancer) and two cancers had k21 values that were below the suspicious range (both $0.01 /$ second). These two cancers (one high grade IDC, one IDC with mucin) were considered suspicious for cancer because of a suspicious irregular mass morphology on MRI and a late phase plateau.

In vivo ${ }^{1} \mathrm{H}$ MRS detected a choline peak in 14 of the 16 cancers (11 IDC, 1 IDC with mucinous features and 1 inflammatory IDC) and no choline peak in two cancers (1 IDC and 1 IDC with associated invasive lobular carcinoma). Based on the ${ }^{1} \mathrm{H}$ MRS findings 14/16 IDC cases were classified as "positive for malignancy," whereas $2 / 16$ of the IDC cases were classified as "negative for malignancy." The resulting positive predictive value of ${ }^{1} \mathrm{H}$ MRS for detection of IDC was $88 \%$.

The invasive tumors with and without detectable choline had a similar distribution of patient ages $(\mathrm{p}<0.85)$ and lesion sizes $(\mathrm{p}<0.76)$. The choline positive tumors ranged in size from 1.5 to $8 \mathrm{~cm}$ (mean $4.1 \mathrm{~cm}$ ) and the two choline-negative cancers were 2.3 and $6 \mathrm{~cm}$ (mean 3.7 $\mathrm{cm}$ ). There was also no significant difference between both groups with regard to morphologic features on MRI. Morphologic analysis of the lesion on MRI showed that 79\% (11/14) of choline positive cancers were masses with spiculated (7/11) or irregular (4/11) borders. The remaining $3 / 14(21 \%)$ of the choline positive cancers were areas of regional enhancement. The morphologic pattern of the two choline-negative cancers was irregular mass and irregular regional enhancement, respectively $(\mathrm{p}<0.80)$. There was no significant difference between the choline positive and negative groups in lesion type (mass versus regional enhancement) on MRI ( $\mathrm{p}<0.26)$.

Enhancement kinetics did differ between both groups; typically both cancers that were cholinenegative showed a late phase plateau (100\%), whereas this was only shown in 5/14 (36\%) of the choline positive cases ( $\mathrm{p}=0.175$, Fischer's exact test). The dominant enhancement pattern seen in $9 / 14(64 \%)$ of the choline positive cancers was a late phase washout of contrast.

Comparison of the choline positive and choline-negative cancers showed that $\mathrm{k} 21$ values differed between both groups and was twice as high in the choline positive cases. The 14 choline positive cancers had $\mathrm{k} 21$ values ranging from 0.01 to $0.20 \mathrm{~s}^{-1}$ (mean $0.083 / \mathrm{second}$ ), whereas the two choline-negative cancers showed $\mathrm{k} 21$ values of 0.03 and $0.05 /$ second, respectively (mean $0.040 /$ second). The choline positive cancers had a higher average $\mathrm{k} 21$ value than the choline-negative cancers but this was not statistically significant $(\mathrm{p}<0.151$ by Mann-Whitney test).

\section{DISCUSSION}

It has been reported by previous studies that MR spectroscopy may be a useful adjunct to breast MR imaging. Reported sensitivities of breast ${ }^{1} \mathrm{H}$ MRS for detection of invasive tumors ranged from $70-100 \%$, and specificities ranges from $67-100 \%$, respectively (9-13). The results of these studies also demonstrated that the choline levels may not be as greatly elevated in some breast cancers as they are in others, with differences in biologic aggressiveness of the tumor as possible explanation $(13,19-21)$. To test this hypothesis our study was designed and focused on the relation between in vivo MRS choline peak data, breast MRI characteristics (both 
morphologic and kinetic), and pharmacokinetic $\mathrm{k} 21$ values as marker of tumor aggressiveness in 16 patients with invasive ductal carcinomas of the breast.

In our study, ${ }^{1} \mathrm{H}$ MRS correctly detected a choline peak in 14/16 invasive breast cancers, resulting in a positive predictive value of $88 \%$. Our study also shows that there is a relation between a positive choline peak identified with ${ }^{1} \mathrm{H}$ MRS and the pharmacokinetic $\mathrm{k} 21$ value of the invasive tumor being imaged. Comparison of choline positive and choline-negative cancers showed that $\mathrm{k} 21$ value was twice as high in the choline positive cases, i.e., $0.083 /$ second versus $0.040 /$ second but this was not statistically significant. $\mathrm{k} 21$ is the pharmacokinetic parameter redistribution rate constant that quantifies the exchange rate of the used MRI contrast agent between the intra- and extravascular spaces (7). It is known from previous studies that the $\mathrm{k} 21$ value is higher in malignant lesions than in benign lesions, because of increased angiogenesis in patients with malignant lesions. Furthermore, the k21 value is related to the amount of angiogenesis and as a consequence can be used as a marker to quantify tumor aggressiveness $(7,16)$. The relation between the presence of choline peak and the $\mathrm{k} 21$ value found in our study is important because it postulates why ${ }^{1} \mathrm{H}$ MRS can be negative in patients with invasive breast cancers. It is possible that invasive tumors need a certain level of aggressiveness to present a choline peak in the ${ }^{1} \mathrm{H}$ MRS spectrum.

This finding is relevant if ${ }^{1} \mathrm{H}$ MRS will be used supplemental to breast MRI with the purpose to replace the number of MRI guided breast biopsies. A recent study by Bartella et al. including 31 malignant and 26 benign breast lesions, reported a $100 \%$ sensitivity (and negative predictive value of $100 \%$ ) of ${ }^{1} \mathrm{H}$ MRS for the detection of cancer (choline peak was present in all 31 malignant lesions studied). They concluded that ${ }^{1} \mathrm{H}$ MRS could be used to reduce the number of MRI-guided biopsies in 58\% without missing any cancer (13). Our results now suggest that false-negative ${ }^{1} \mathrm{H}$ MRS results are not only caused by patient motion or inhomogeneities of the magnetic field as has been reported by others, but can also occur in less aggressive invasive breast tumors with low $\mathrm{k} 21$ values.

Besides the pharmacokinetic $\mathrm{k} 21$ value, the MRI contrast-enhancement kinetics differed between choline positive and choline-negative cancers. Whereas both choline-negative cancers showed initial rapid wash-in of contrast followed by a plateau phase (100\%), this pattern was only seen in $36 \%$ of the choline positive cancers. The dominant enhancement pattern in choline positive cancers, seen in $64 \%$, was initial rapid wash-in of contrast followed by washout. This difference in enhancement pattern suggests that choline-negative cancers may be less aggressive. It is known from previous studies that an enhancement curve marked by rapid washin followed by washout of contrast is very specific for a malignant lesion (with occasional benign findings such as lymph nodes or papillomas which have marked vascularity), whereas the an enhancement curve marked by rapid wash-in followed by a plateau phase, is seen in both benign and malignant lesions $(16,18)$.

Morphologic features of the tumor analyzed with MRI of the breast were not related to presentation of a choline peak on ${ }^{1} \mathrm{H}$ MRS examination. Furthermore, we found no relation between tumor size and the result of ${ }^{1} \mathrm{H}$ MRS. Previously, it has been hypothesized that positive MRS studies would more likely occur in larger tumors and that a negative MRS exam would more likely occur in small tumors, which might not be as accurately targeted by the MRS voxel or which might have fewer tumor cells to produce a choline peak per voxel $(22,23)$. However, in our study, tumor size did not differ between choline-positive and choline-negative tumors. With choline-positive tumors ranging in size from 1.5 to $8.0 \mathrm{~cm}$ (mean $4.1 \mathrm{~cm}$ ) and cholinenegative tumors ranging in size from 2.3 to $6.0 \mathrm{~cm}$ (mean $3.7 \mathrm{~cm}$ ). This is in agreement with findings of a recent study that concluded that choline levels are probably more related to the malignant nature of the tumor than to the tumor size (13). 
In this study, we a positive choline peak was defined, if after baseline corrections, there was a well-defined peak at $3.2 \mathrm{ppm}$ greater than two times the standard deviation of the baseline noise as determined from a peak-free region of the spectrum. The main advantage of measurement of choline signal relative to noise is that it is accurate and allows fast image analysis, which is important when ${ }^{1} \mathrm{H}$ MRS is included in clinical imaging protocols for breast cancer. To optimize the accuracy of ${ }^{1} \mathrm{H}$ MRS we included breast lesions $>1 \mathrm{~cm}$ in diameter, so partial volume averaging due to small tumor volume within voxel and/or alignment of the voxel over areas of normal tissue, and as a consequence negative choline detection, is less likely to occur. Because mean tumor size between choline-positive and negative cancers did not differ in our study, we do not believe that partial volume averaging played a role.

Our study also has limitations, specifically the relatively small number of patients that were included, which decreased the power of the study. However, our study indicates a direction for future investigations of k21 and MRS findings, within a larger series. Furthermore, because only malignant lesions were included, no conclusions could be made about the specificity or diagnostic accuracy of MRI and MRS combined, because of the absence of a benign comparison group.

In conclusion, ${ }^{1} \mathrm{H}$ MRS correctly identified a choline peak in 14/16 (88\%) of the invasive ductal breast cancers. A false-negative ${ }^{1} \mathrm{H}$ MRS study occurred in two patients with invasive ductal breast cancer. The choline-negative cancers presented lower pharmacokinetic $\mathrm{k} 21$ values on contrast-enhanced MRI of the breast. The study results indicate that false-negative ${ }^{1} \mathrm{H}$ MRS exams do occur in breast cancer patients, and that the presence of a choline peak on ${ }^{1} \mathrm{H}$ MRS as malignancy marker is related to the $\mathrm{k} 21$ value of the invasive tumor being imaged.

\section{REFERENCES}

1. Orel SG, Schnall MD. MR imaging of the breast for detection, diagnosis, and staging of breast cancer. Radiology 2001;220:13-30. [PubMed: 11425968]

2. Robson ME, Offit K. Breast MRI for women with hereditary cancer risk. JAMA 2004;292:1368-70. [PubMed: 15367560]

3. Kriege M, Brekelmans CT, Boetes C, et al. Efficacy of MRI and mammography for breast-cancer screening in woman with familial or genetic predisposition. N Engl J Med 2004;351:427-37. [PubMed: 15282350]

4. Daniel BL, Yen YF, Glover GH, et al. Breast disease: dynamic spiral MR imaging. Radiology 1998;209:499-509. [PubMed: 9807580]

5. Liberman L, Morris EA, Lee MJ, et al. Breast lesions detected on MR imaging: features and positive predictive value. AJR Am J Roentgenol 2002;179:171-8. [PubMed: 12076929]

6. Agoston AT, Daniel BL, Herfkens RJ, et al. Intensity-modulated parametric mapping for simultaneous display of rapid dynamic and high-spatial-resolution breast MR imaging data. Radiographics 2001;21:217-26. [PubMed: 11158656]

7. Mariano MN, Van den Bosch MAAJ, Daniel BL, et al. Contrast-enhanced MRI of ductal carcinoma in situ: Characteristics of a new intensity-modulated parametric mapping technique correlated with hsitopathologic findings. J Magn Reson Imaging 2005;22:520-6. [PubMed: 16142701]

8. Robinson SP, Barton SJ, McSheehy PM, Griffiths JR. Nuclear magnetic resonance spectroscopy of cancer. Br J Radiol 1997;70:S60-9. [PubMed: 9534719]

9. Jacobs MA, Barker PB, Argani P, et al. Combined dynamic contrast enhanced breast MR and proton spectroscopic imaging: a feasibility study. J Magn Reson Imaging 2005;21:23-8. [PubMed: 15611934]

10. Stanwell P, Gluch L, Clark D, et al. Specificity of choline metabolites for in vivo diagnosis of breast cancer using ${ }^{1} \mathrm{H}$ MRS at 1.5T. Eur Radiol 2005;15:1037-43. [PubMed: 15351906]

11. Bolan PJ, Nelson MT, Yee D, Garwood M. Imaging in breast cancer: Magnetic resonance spectroscopy. Breast Cancer Res 2005;7:149-52. [PubMed: 15987466] 
12. Jagannathan NR, Kumar M, Seenu V. Evaluation of total choline from in-vivo volume localized proton MR spectroscopy and its response to neoadjuvant chemotherapy in locally advanced breast cancer. Br J Cancer 2001;84:1016-22. [PubMed: 11308247]

13. Bartella L, Morris EA, Dershaw DD, et al. Proton MR spectroscopy with choline peak as malignancy marker improves positive predictive value for breast cancer diagnosis: preliminary study. Radiology 2006;239:686-92. [PubMed: 16603660]

14. Castillo M, Kwock L. Clinical applications of proton magnetic resonance spectroscopy in the evaluation of common intracra-nial tumors. Top Magn Reson Imaging 1999;10:104-13. [PubMed: 10551625]

15. Tse GM, Cheung HS, Pang LM, et al. Characterization of lesions of the breast with proton MR spectroscopy: comparison of carcinomas, benign lesions, and phyllodes tumors. AJR Am J Roentgenol 2003;181:1267-72. [PubMed: 14573418]

16. Knopp MV, Weiss E, Sinn HP, et al. Pathophysiologic basis of contrast enhancement in breast tumors. J Magn Reson Imaging 1999;10:260-6. [PubMed: 10508285]

17. Star-Lack J, Nelson SJ, Kurhanewicz J, Huang LR, Vigneron DB. Improved water and lipid suppression for 3D PRESS CSI using RF band selective inversion with gradient dephasing (BASING). Magn Reson Med 1997;38:311-21. [PubMed: 9256113]

18. Ikeda DM, Hylton NM, Kinkel K, et al. Development, standardization, and testing of a lexicon for reporting contrast-enhanced breast magnetic resonance imaging studies. J Magn Reson Imaging 2001;13:889-95. [PubMed: 11382949]

19. Yeung DK, Yang WT, Tse GM. Breast cancer: in vivo proton MR spectroscopy in the characterization of histopathologic subtypes and preliminary observations in axillary node metastases. Radiology 2002;225:190-7. [PubMed: 12355004]

20. Gribbestad IS, Singstad TE, Nilsen G, et al. In vivo 1H MRS of normal breast and breast tumors using a dedicated double breast coil. J Magn Reson Imaging 1998;8:1191-7. [PubMed: 9848727]

21. Merchant TE. MR spectroscopy of the breast. Magn Reson Imaging Clin N Am 1994;2:691-703. [PubMed: 7489317]

22. Kvistad KA, Bakken IJ, Gribbenstad IS, et al. Characterization of neoplastic and normal human breast tissues with in vivo ${ }^{1} \mathrm{H}$ MR spectroscopy. J Magn Reson Imaging 1999;10:159-64. [PubMed: 10441019]

23. Yeh ED. Characterization of breast lesions with proton MR spectroscopy. AJR Am J Roentgenol 2003;181:1273-4. [PubMed: 14573419] 
Table 1

Baseline Characteristics of the Study Population

\begin{tabular}{rrlll}
\hline Case & Age & Mammography & Ultrasound & Pathology \\
\hline 1 & 43 & No abnormalities & NA & IDC with ILC \\
2 & 38 & $2.5 \mathrm{~cm}$ spic. mass & NA & IDC \\
3 & 26 & Scar & $1.2 \mathrm{~cm}$ round mass & IDC \\
4 & 39 & $6.0 \mathrm{~cm}$ indistinct mass + calcs & $1.2 \mathrm{~cm}$ heterog. mass & IDC \\
5 & 50 & $4.0 \mathrm{~cm}$ indistinct mass + calcs & $1.5 \mathrm{~cm}$ oval mass & IDC \\
6 & 53 & No abnormalities & $2.9 \mathrm{~cm}$ irreg. mass & IDC \\
7 & 51 & $1.5 \mathrm{~cm}$ spic. mass + calcs & NA & IDC \\
8 & 43 & $4.0 \mathrm{~cm}$ indistinct mass + calcs & $3.0 \mathrm{~cm}$ irreg. mass & IDC \\
9 & 51 & $1.5 \mathrm{~cm}$ spic. mass + calcs & $1.5 \mathrm{~cm}$ heterog. mass & IDC \\
10 & 47 & $1.2 \mathrm{~cm}$ lobulated mass + calcs & $2.5 \mathrm{~cm}$ microlob. mass & IDC with mucin \\
11 & 36 & $3.5 \mathrm{~cm}$ spic. mass + calcs & NA & IDC \\
12 & 43 & $1.8 \mathrm{~cm}$ indistinct mass + calcs & $3.3 \mathrm{~cm}$ irregular mass & IDC \\
13 & 55 & $3.5 \mathrm{~cm}$ spic. mass & NA & IDC \\
14 & 79 & $8.0 \mathrm{~cm}$ irreg. mass & Irreg. shadow, edematous & IDC-inflammatory \\
15 & 53 & $4.5 \mathrm{~cm}$ indistinct mass & Ill-defined mass & IDC \\
16 & 48 & $6.5 \mathrm{~cm}$ calcs. only & $3.6 \mathrm{~cm}$ lobulated mass & IDC \\
\hline
\end{tabular}

IDC, invasive ductal carcinoma; ILC, invasive lobular carcinoma; NA, not applicable; spic, spiculated; lob, lobulated; calcs, calcifications; irreg, irregular; heterog., heterogeneous; homog., homogeneous. 


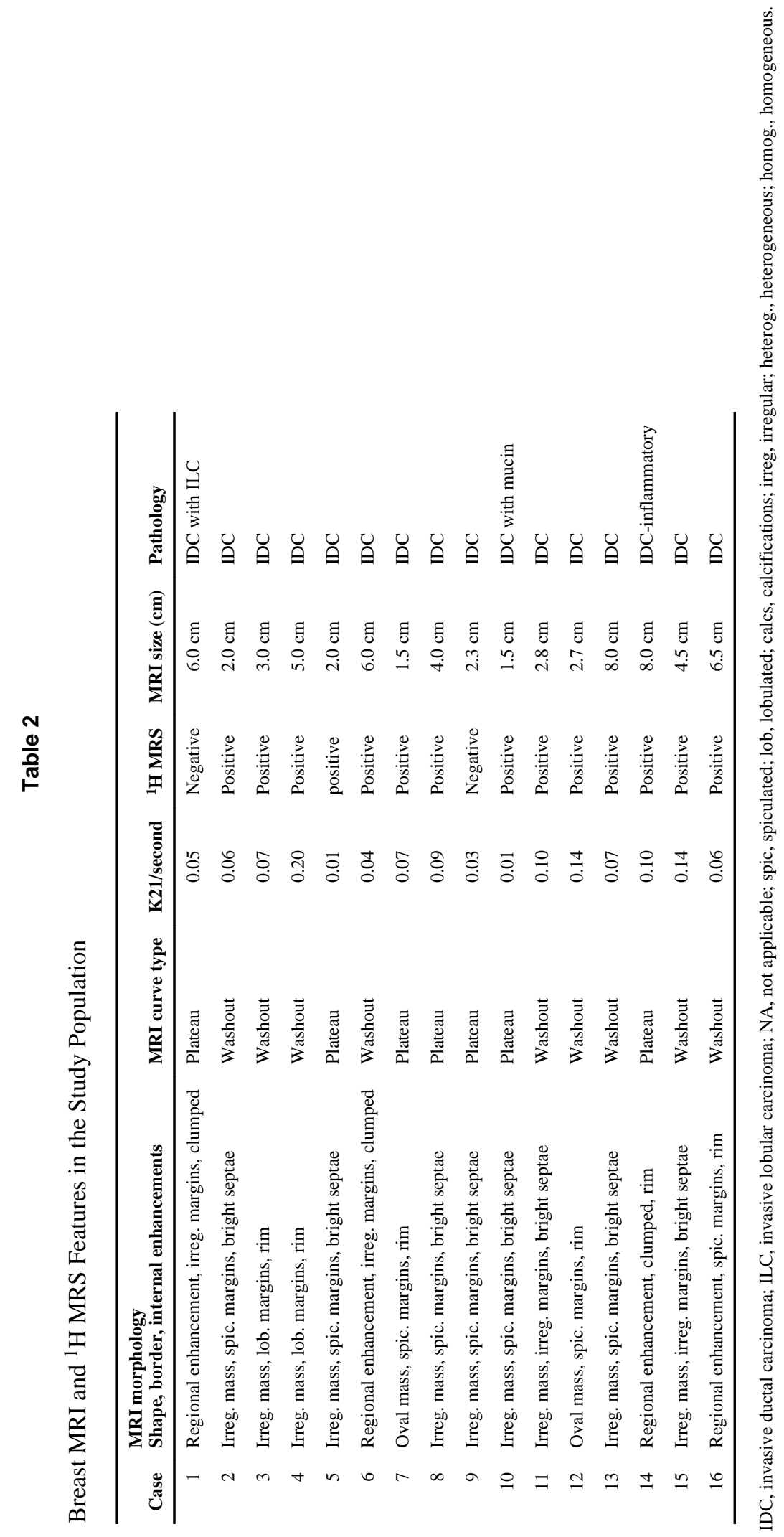

Breast J. Author manuscript; available in PMC 2010 March 20. 